\title{
Family therapy experience in the training of general psychiatrists
}

\author{
Philip Wilkinson, formerly Senior House Officer in Child and Adolescent Psychiatry; \\ and PIETER van Boxel, Consultant in Child and Adolescent Psychiatry, Family and \\ Young Persons Unit, Paxton House, 57 Bath Road, Reading RG3 2BA
}

This paper describes the work of a family therapy team which includes a senior house officer, or registrar, and discusses how such experience is of value to the trainee in general adult psychiatry.

\section{The trainer's perspective}

During a six month placement in child and adolescent psychiatry a trainee from the Oxfordshire Regional SHO/Registrar Training Scheme joins a consultant, senior registrar, and senior psychiatric social worker in a weekly family therapy clinic.

Patients with problems compatible with this treatment setting are allocated from general practitioner or paediatric referrals. At the initial assessment the combination of therapist-co-therapist is considered by the team with regard to the needs of the patient, the type and degree of difficulty of the problem, and the relative experience of the trainee; the trainee participates in the majority of assessments. Other team members participate through video and telephone link and the whole team remains involved in the continuing work with the family at future follow-up sessions.

Experience has shown that such active participation in this small team provides a good learning opportunity for the junior trainees who are well able to respond to the challenge and can add their own valuable contribution to the team's work.

\section{The trainee's experience}

Trainees receive basic teaching through seminars in family systems theory and the major family therapy paradigms; specific assessment skills and therapeutic interventions are demonstrated in treatment sessions by the senior co-therapist and set against a broad theoretical base.

With increasing experience the trainee can use newly acquired techniques as well as drawing on other more familiar treatment approaches. The trainee must adapt to joint interviewing, hypothesising and formulating problems and providing interventions in systemic terms; this task is facilitated by the process of immediate feedback and case discussion that such a setting provides. Easy integration is achieved into this experienced clinical team in a way that allows the presence of a less experienced and generally younger team member to be acceptable to client families.

In the course of the placement trainees encounter a variety of clinical scenarios, ranging from families adapting to external stresses or bereavement to those coping with a severely disturbed youngster; some particularly interesting cases have included children experiencing fatigue and other chronic somatic symptoms.

\section{Comments}

Experience in child and adolescent psychiatry is important for any psychiatrist in that it offers insight into the development of personality and psychological disturbance; working in a family therapy clinic gives a unique perspective on the functioning of adults as parents and the consequences within families of psychiatric illness.

New diagnostic and therapeutic techniques are acquired. Trainees learn the principles of systems theory, for example, the nature of circular causality and the impact of the therapist within the system, along with therapeutic techniques such as the use of positive connotation and circular questioning to promote change.

Outside the family therapy clinic there is potentially a wide application for these skills in interviewing families to help unravel aetiological processes in adult mental illness and in recruiting and reinforcing family support systems. Recognising this, trainers at McGill University in Montreal have incorporated a two-year family therapy training programme into the psychiatric residency for many years (Guttman et al, 1990). The training is mandatory and usually takes place during placements in adult specialties.

What might be the lasting impact of such experience? Trainees in Wisconsin receive a six month programme in basic marital and family therapy. From a questionnaire survey, Carter (1986) concluded that this programme had an enduring effect on psychiatrists' style of work and their readiness to refer complex cases on to formal family therapy agencies. 
In the United Kingdom, the Royal College of Psychiatrists (1988) recommends that the training of general psychiatrists should include a placement in child and adolescent psychiatry with "exposure to a variety of theoretical orientations and exponents of different sorts of treatment including individual and family therapy".

It is not clear, however, to what extent trainees nationally have access to basic family therapy training and whether this experience receives appropriate emphasis. Given its wide application, is there not a case for further family therapy training during placements in general adult and old age psychiatry? As services expand within the community it is expedient that psychiatrists not only treat individuals but are also equipped to work with whole families.

\section{Conclusions}

The trainee in general psychiatry is most likely to encounter family therapy during a placement in child and adolescent psychiatry. A small and experienced clinical team is able to offer effective basic training, fostering an understanding of systems theory and the acquisition of important skills for working with families; since such skills are of increasing value to the general psychiatrist, we believe experience in family therapy should be an essential component in any training scheme.

\section{References}

CARTER, R. E. (1986) An evaluation of a partial training program in psychiatric residency on the later practice of marital and family therapy. American Journal of Family Therapy, 14, 145-153.

Guttman, H., Feldman, R. \& Bravermans, S. (1990) An integrated approach to family therapy training for psychiatric residents. Canadian Journal of Psychiatry, 35, 58-63.

Royal College of Psychiatrists Executive Committee of the Child and Adolescent Specialist Section (1988) Guidelines for the training of general psychiatrists in child and adolescent psychiatry. Bulletin of the Royal College of Psychiatrists, 12, 391-392.

\section{Joint RCPsych/AUTP Workshop on Psychiatry in the Undergraduate Medical Curriculum}

A joint RCPsych/AUTP Workshop on Psychiatry in the Undergraduate Medical Curriculum will be held from 1.30 p.m. to 5.00 p.m. on Monday 25 January 1993 at the Society of Chemical Industry, 14/15 Belgrave Square, London SW 1 .

Speakers will include Professor David Shaw (GMC Education Committee), Professor Chris
Thompson, Dr Jan Scott and Dr Phillip Snashell (Sub-Dean, Charing Cross and Westminster Medical School).

Further details and an application form can be obtained from Mrs Jennifer Andrews, Education Department, Royal College of Psychiatrists, 17 Belgrave Square, London SW1X 8PG. 